\title{
AVIFAUNA COMO BIOINDICADORA PARA AVALIAÇÃO DA RESTAURAÇÃO FLORESTAL: ESTUDO DE CASO EM UMA FLORESTA RESTAURADA COM 40 ANOS EM VIÇOSA - MG
}

\author{
AVIFAUNA AS A BIOINDICATOR FOR THE EVALUATION OF FOREST RESTORATION: A \\ CASE STUDY IN A 40-YEAR OLD RESTORED FOREST IN VIÇOSA, MINAS GERAIS STATE
}

\author{
Graziele Hernandes Volpato ${ }^{1}$ Aurino Miranda Neto ${ }^{2}$ Sebastião Venâncio Martins ${ }^{3}$
}

\begin{abstract}
RESUMO
O método ponto de escuta foi utilizado com o objetivo de avaliar a comunidade de aves em um pequeno trecho de 1 ha de floresta restaurada com mais de 40 anos de implantação, no município de Viçosa - MG. Um total de 20 pontos foi amostrado no período reprodutivo das aves no ano de 2011. Foram registrados 31 espécies de aves e 160 contatos, sendo a maioria das espécies típicas da Floresta Estacional Semidecidual e cinco espécies endêmicas do Bioma Mata Atlântica. No presente estudo, a presença de aves consumidora de frutos e de aves insetívoras de sub-bosque que apresentam pouca tolerância a ambientes abertos e de forte insolação, sugere a boa qualidade do habitat amostrado, confirmando que a floresta restaurada se encontra em estádio avançado de sucessão, conforme estudos anteriores. Os resultados do presente estudo também demonstram que mesmo em uma área pequena e com poucos dias de amostragens foi possível obter uma rápida caracterização da comunidade de aves e, a partir dessa composição, relacioná-la com as condições do habitat, permitindo uma avaliação segura do sucesso de um projeto de restauração florestal.
\end{abstract}

Palavras-chave: aves frugívoras; aves insetívoras; sub-bosque; Mata Atlântica; recuperação ambiental.

\begin{abstract}
The point-counts method was used to evaluate the bird community in a small patch of forest ( 1 ha) restored through reforestation, with more than 40 years of deployment, in Viçosa, MG state. In total, 20 points were sampled in the reproductive period of the birds, during 2011. A total of 31 bird species and 160 contacts were recorded, and all species were identified as typical from a semi-deciduous forest and included five endemic species of the Atlantic forest biome. In the present study, the presence of frugivorous and insectivorous birds in the understory with little tolerance for open environments and strong insolation, suggests the good quality of the sampled habitat, confirming that the restored forest is in an advanced stage of succession. The results of this study also demonstrate that even in a small area, and with a few days of sampling, it is possible to obtain a rapid characterization of the bird community, being possible to associate it to the habitat conditions and allowing a safe evaluation of the success of a forest restoration project.

Keywords: Frugivores; insectivores; understory; Atlantic forest; environmental recovery.
\end{abstract}

\section{INTRODUÇÃO}

Com o desaparecimento e o isolamento das áreas de florestas nativas, a recuperação de áreas degradadas pode ser uma importante ferramenta para refazer comunidades florestais e estabelecer corredores entre fragmentos vegetacionais (MARTINS, 2009a; RODRIGUES et al., 2009). Entretanto,

1 Bióloga, Dra., Pós-doutorado em Ciência Florestal, Departamento de Engenharia Florestal, Universidade Federal de Viçosa, Av. P. H. Rolfs, s/n, Campus Universitário, CEP 36570-000, Viçosa (MG), Brasil. gravolpato@yahoo. com.br

2 Engenheiro Florestal, Dr., Pós-graduado em Ciência Florestal pelaUniversidade Federal de Viçosa, Av. P. H. Rolfs, s/n, Campus Universitário, CEP 36570-000, Viçosa (MG), Brasil. aur.neto@gmail.com

3 Engenheiro Florestal, Dr., Professor do Departamento de Engenharia Florestal, Universidade Federal de Viçosa, Av. P. H. Rolfs, s/n, Campus Universitário, CEP 36570-000, Viçosa (MG), Brasil. venancio@ufv.br

Recebido para publicação em 19/08/2014 e aceito em 16/11/2016

Ci. Fl., v. 28, n. 1, jan.- mar., 2018 
além da restauração ambiental de uma área, a avaliação e o monitoramento desta também é fundamental para indicar o sucesso da restauração ou mesmo da necessidade de correções (RODRIGUES et al., 2009). Diversos indicadores de avaliação e monitoramento da restauração florestal têm sido utilizados, como a produção de serapilheira (ARATO; MARTINS; FERRARI, 2003), a composição florística e estrutura da vegetação (ARAUJO et al., 2005; 2006), a cobertura de copas/fechamento do dossel (MELLO; MIRANDA; DURIGAN, 2007); o banco de sementes do solo (MARTINS et al., 2008; MARTINS, 2009b), entre outros.

Assim, quase que a totalidade dos indicadores de avaliação é oriunda da vegetação (DURIGAN; ENGEL, 2012) e apesar de ser destacada como importante, a fauna ainda é pouco utilizada para este fim (VOLPATO et al., 2012; PADOVEZI; RODRIGUES; HORBACH, 2014). A fauna, em áreas restauradas ou em processo de restauração, atua em diferentes processos ecológicos, como polinização, dispersão e predação de sementes e, portanto, são importantes elementos para uma restauração autossustentável (VOLPATO et al., 2012). Dentro desse contexto, as aves são consideradas como bom táxon para avaliar e monitorar as consequências ecológicas de mudança de ambiente (LAWTON, 1996; VOLPATO et al., 2012; PADOVEZI; RODRIGUES; HORBACH, 2014), como a restauração de uma área degradada, por apresentarem alta especificidade quanto ao seu território e habitat, o que pode ser relacionado a diferentes estágios sucessionais da vegetação (VOLPATO et al., 2012).

Diversos autores demonstram que a restauração florestal de áreas degradadas proporciona um aumento na biodiversidade, principalmente para a avifauna (NICHOLS; GRANT, 2007; MUNRO et al., 2010; VOLPATO et al., 2012; PADOVEZI; RODRIGUES; HORBACH, 2014). Munro et al. (2009) descrevem que, em áreas restauradas, a complexidade da estrutura da vegetação aumenta com o avanço da idade. E essa maior complexidade é apontada por Munro et al. (2010) como uma importante característica para a presença de espécies de aves em restauração. Assim, espera-se uma relação direta da composição das avifauna com o estágio sucessional de uma área restaurada ou em processo de restauração, uma vez, que as aves interagem com a estrutura e composição do habitat (VOLPATO et al., 2012; PADOVEZI; RODRIGUES; HORBACH, 2014).

O presente estudo teve o objetivo de descrever a comunidade de aves em uma floresta restaurada com mais de 40 anos de implantação, considerando o hábito alimentar, o estrato preferencial de forrageamento e a sensibilidade das aves a perturbações do habitat. Com os resultados do presente estudo, procura-se também indicar o potencial da avifauna para a avaliação do sucesso de restauração desta floresta.

\section{MATERIAL E MÉTODOS}

\section{Área de estudo}

O estudo foi desenvolvido em um trecho de floresta restaurada, localizado no campus da Universidade Federal de Viçosa, Viçosa - MG $\left(20^{\circ} 45^{\prime} 14^{\prime \prime}\right.$ 'S e $\left.42^{\circ} 45^{\prime} 53^{\prime \prime W}\right)$. Uma área com pastagem abandonada foi restaurada a partir de reflorestamento com plantios de mudas de espécies nativas e exóticas entre os anos de 1967 e 1969, pelo setor de Silvicultura da Universidade Federal de Viçosa. Foram feitos plantios heterogêneos no espaçamento de 4,0 x 5,0 m, em uma área de 1 ha, em que foi plantado um total de 485 indivíduos e 56 espécies (MIRANDA NETO et al., 2012b). Este trecho de floresta restaurada é contínuo a plantios homogêneos de Pinus sp., Terminalia sp., Araucaria angustifolia (Bertol.) Kuntze, Chlorophora excelsa (Welw.) Benth., e por fragmentos florestais secundários da fitofisionomia Floresta Estacional Semidecidual, que totalizam aproximadamente 35 hectares, especialmente a "Mata da Silvicultura", vizinha à floresta restaurada (cerca de 100 metros de distância). A "Mata da Silvicultura" possui 17 ha (MEIRA NETO; MARTINS, 2002) e encontra-se protegida de corte e extração de madeira desde o ano de 1936 (MARISCAL-FLORES, 1993). Atualmente o estrato arbustivo-arbóreo adulto (DAP $\geq 5,0 \mathrm{~cm}$ ) da floresta restaurada possui altura média de 10,6 m, DAP médio de $15 \mathrm{~cm}$ e área basal de 47,8 $\mathrm{m}^{2}$ ha-1 (MIRANDA NETO et al., 2012b). A vegetação desse trecho de floresta restaurada foi classificada por Miranda Neto et al. (2012a) em estádio avançado de sucessão. Miranda Neto et al. (2012a; 2012b) estimaram em 1 ha dessa floresta restaurada 1.432 indivíduos e 112 espécies arbustivo-arbóreas no estrato adulto (DAP $\geq 5,0 \mathrm{~cm}$ ) e 24.225 indivíduos e 102 espécies arbustivo-arbóreas no estrato de regeneração natural (altura $\geq 0,50 \mathrm{~m}$ e $\mathrm{DAP} \leq 5,0 \mathrm{~cm}$ ). No estrato adulto, 178 indivíduos e 19 espécies foram provenientes do plantio e o restante 
foi originário do banco de sementes ou oriundas de propágulos vindos de fragmentos de Floresta Estacional Semidecidual das proximidades (MIRANDA NETO et al., 2012b).

\section{Amostragens de Aves}

Para obtenção de dados quantitativos das populações de aves neste trecho de 1 ha de floresta restaurada foi utilizado o método de amostragem por pontos com raio limitado de $25 \mathrm{~m}$ (BIBBY; BURGESS; HILL, 1992). As amostragens consistiram em contar o número de contatos com cada casal ou bando de espécies de aves que vocalizou em um espaço de tempo padrão de 15 minutos em cinco pontos previamente determinados, distantes $100 \mathrm{~m}$ um do outro. As amostragens se iniciaram a partir do amanhecer (em torno das $6 \mathrm{~h}$ ) e finalizadas após aproximadamente três horas, quando as atividades das aves diminuem significativamente (BIBBY; BURGESS; HILL, 1992). Cada par ou grupo de espécies de aves foi considerado como um contato durante a amostragem em um ponto. Em cada manhã, um conjunto de cinco pontos foi amostrado, representando um dia de amostragem. Foram amostradas quatro manhãs em dezembro de 2011, período considerado como favorável para registro de aves na Floresta Estacional Semidecidual (VOLPATO et al., 2009), totalizando assim 20 pontos amostrais.

\section{Análise dos dados}

Foi verificado se o esforço amostral realizado foi suficiente para amostrar um número representativo de espécies de aves no trecho de floresta restaurada. Para isso, uma curva de rarefação foi construída com o número de espécies (Mao Tau) e o número de amostragens. O estimador Jackknife 1 foi utilizado para calcular a riqueza estimada de espécies. Em ambas as análises foi utilizado o programa EstimateS 8.0.0 (COLWELL, 2006).

Os hábitos alimentares de cada espécie registrada foram determinados considerando-se observações de campo e informações da literatura (ANJOS, 2001; VOLPATO; PRADO; ANJOS, 2010) e categorizadas como: frugívoros (consumo preferencial de polpa de frutos), frugívoro/insetívoros (consumo de polpa de frutos, sementes, folhas e eventualmente pequenos invertebrados), insetívoros (consumo preferencial de invertebrado). As espécies de aves também foram classificadas conforme o estrato preferencial para o forrageamento, conforme Parker, Stotz, Fitzpatrick (1996) em: espécies de sub-bosque (espécies que forrageiam principalmente nos estratos mais inferiores da floresta, do solo até $7 \mathrm{~m}$ ) e espécies de copa (espécies que procuram seu alimento e/ou presa nas copas da floresta). Baseado em Parker, Stotz, Fitzpatrick (1996), cada espécie de ave foi classificada quanto ao grau de sensibilidade a perturbações do habitat. Foram também identificadas as espécies endêmicas à Mata Atlântica, conforme Bencke et al. (2006). A lista de classificação das espécies de aves seguiu Comitê Brasileiro de Registros Ornitológicos (PIACENTINI et al., 2015).

\section{RESULTADOS E DISCUSSÃO}

Foram registrados 31 espécies de aves e 204 contatos no trecho de floresta restaurada amostrada (Tabela 1). A riqueza de espécies registradas no presente estudo foi baixa quando comparada à riqueza de aves de outras florestas nativas da região (RIBON; SIMON; DE MATTOS, 2003). Contudo, a curva de acumulação de espécies apresentou uma leve inclinação (Figura 1), indicando a tendência à assíntota. Considerando o estimador de riqueza Jackknife, o levantamento observado atingiu 84,5\% da riqueza das espécies esperados, indicando a possibilidade de novos registros de espécies, como indica a curva do estimador de riqueza Jackknife (Figura 1). Anjos (2007) demonstrou que quatro manhãs de amostragem utilizando o método por pontos foi eficiente para o registro de uma proporção considerável de espécies de aves em Floresta Estacional Semidecidual. Assim, o baixo número de espécies registrado no presente estudo pode ser uma consequência da pequena área amostral avaliada. 


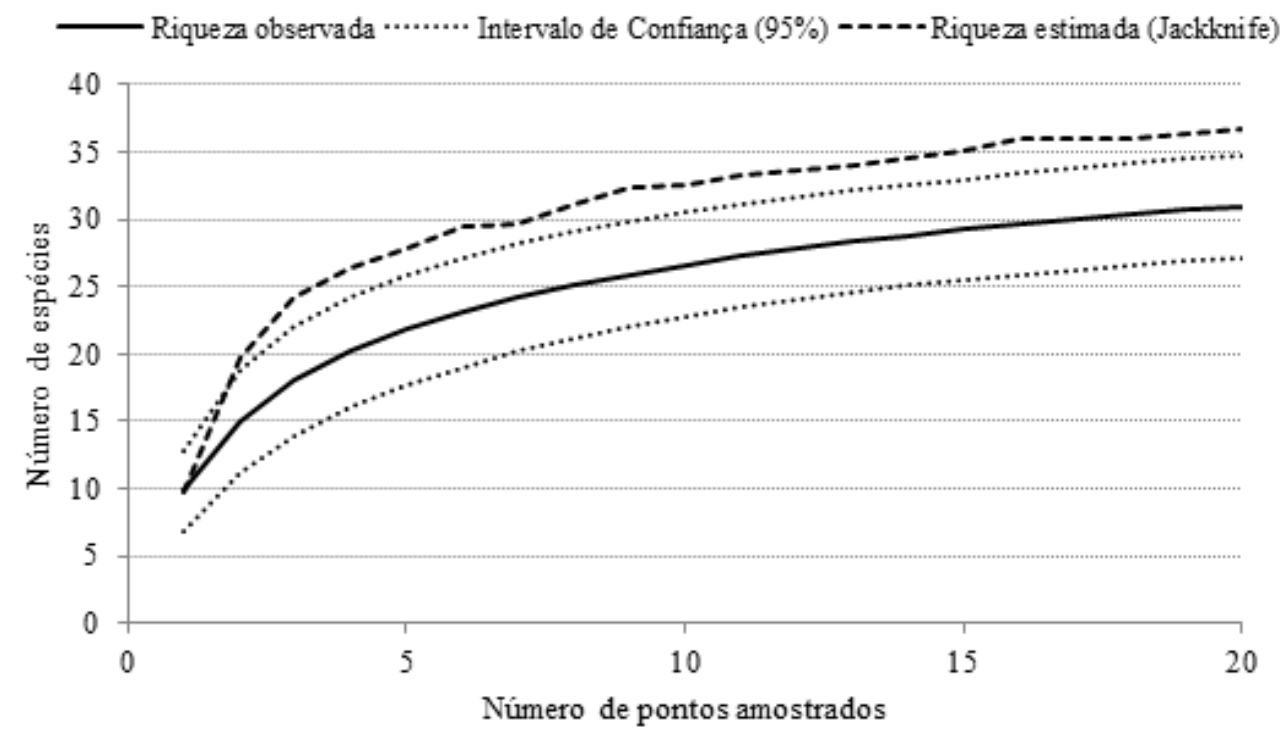

FIGURA 1: Curva de espécies acumuladas (riqueza observada) com o intervalo de confiança e número de espécies estimadas pelo Jackniffe 1 de aves registradas em um trecho de floresta restaurada com 40 anos em Minas Gerais.

FIGURE 1: Curve of accumulated species (observed richness) with the confidence interval and number of species estimated by Jackniffe 1 of birds recorded in a section of a 40-year old restored forest in Minas Gerais state.

Apesar da baixa riqueza de espécies, a avaliação da composição das espécies revela que a floresta restaurada com mais de 40 anos foi formada por espécies de aves tipicamente de Floresta Estacional Semidecidual (RIBON; SIMON; DE MATTOS, 2003), incluindo seis espécies endêmicas do Bioma Mata Atlântica: Veniliornis spilogaster, Chiroxiphia caudata, Mionectes rufiventris, Myiornis auricularis, Tangara cyanoventris e Hemithraupis ruficapilla (Tabela 1). A importância das espécies endêmicas é com frequência apontada como uma estratégia na manutenção da biodiversidade (PIMM et al., 1995; BROOKS; BALMFORD, 1999). Contudo, Antunes (2007) mostrou que diversas aves endêmicas da Mata Atlântica, especialmente para aquelas não obrigatoriamente florestais-dependentes como as cinco espécies identificadas no presente estudo, podem estar se beneficiando com a fragmentação e a perturbação ambiental. Assim, aves com maior plasticidade na utilização do habitat podem adaptar-se facilmente a um novo ambiente. Dessa maneira, a utilização das aves endêmicas na avaliação dos processos de restauração pode não ser um bom indicador, pelo menos quando usado de forma isolada.

No presente estudo, a maioria das aves registradas (64,5\%) possui baixa sensibilidade aos efeitos de perturbações ambientais, enquanto outras 32,3\% possuem sensibilidade média. Apenas Habia rubica possui alta sensibilidade a perturbações ambientais (Tabela 2). Entre as aves que apresentaram sensibilidade média e alta, a maior parte são aves insetívoras de sub-bosque, como Mionectes rufiventris, Habia rubica, Chiroxiphia caudata, Lathrotriccus euleri, Corythopis delalandi e Basileuterus culicivorous (Tabela 1). Os insetívoros de sub-bosque são um grupo de aves com requisitos ecológicos mais específicos, especializado em forragear em determinados estratos e substratos da vegetação e com pouca tolerância à insolação (ALEIXO; VIELLIARD, 1995; CHRISTIANSEN; PITTER, 1997; GALLETI; ALEIXO, 1998; ALEIXO, 2001; VOLPATO et al., 2006). 
TABELA 1: Lista de espécies de aves e número de contatos $(\mathrm{N})$ registradas em floresta restaurada com 40 anos em Minas Gerais, considerando o estrato de forrageio (SUB - sub-bosque; COP - Copa), hábito alimentar (INS - Insetívoros; FRU/INS - Frugívoros/Insetívoros; FRU - Frugívoros) e grau de sensibilidade a perturbações do habitat (A - Alta, M - Média, B - baixa). * Indica espécie endêmica ao Bioma Mata Atlântica.

TABLE 1: $\quad$ Bird species and number of contacts $(\mathrm{N})$ recorded in a 40-year old restored forest in Minas Gerais state, considering the feeding behavior (SUB- sub-canopy, COP-canopy), the feeding habits (INS insectivores, FRU/INS- frugivores/ insectivores, FRU- frugivores), and the sensitivity to disturbance (A- high, M- Medium, B- Low). * indicate bird species endemic to the Atlantic Forest.

\begin{tabular}{|c|c|c|c|c|c|}
\hline Espécies & Nome popular & $\mathrm{N}$ & $\begin{array}{l}\text { Estrato de } \\
\text { forrageio }\end{array}$ & $\begin{array}{l}\text { Hábito } \\
\text { alimentar }\end{array}$ & Sensibilidade \\
\hline Penelope obscura & jacuaçu & 2 & $\mathrm{COP}$ & FRU & M \\
\hline Patagioenas picazuro & asa-branca & 2 & $\mathrm{COP}$ & FRU/INS & M \\
\hline Tapera naevia & saci & 1 & SUB & INS & $\mathrm{B}$ \\
\hline Glaucidium brasilianum & caburé & 1 & $\mathrm{COP}$ & INS & B \\
\hline Pteroglossus aracari & araçari-de-bico-branco & 5 & $\mathrm{COP}$ & FRU & M \\
\hline Veniliornis spilogaster* & picapauzinho-verde-carijó & 4 & $\mathrm{COP}$ & INS & M \\
\hline Milvago chimachima & carrapateiro & 1 & $\mathrm{COP}$ & INS & B \\
\hline Thamnophilus caerulescens & choca-da-mata & 3 & SUB & INS & B \\
\hline Synallaxis spixi & joão-teneném & 1 & SUB & INS & B \\
\hline Chiroxiphia caudata* & tangará & 12 & SUB & FRU/INS & B \\
\hline Mionectes rufiventris* & abre-asa-de-cabeça-cinza & 6 & SUB & INS & M \\
\hline Corythopis delalandi & estalador & 13 & SUB & INS & M \\
\hline Tolmomyias sulphurescens & bico-chato-de-orelha-preta & 17 & $\mathrm{COP}$ & INS & M \\
\hline Myiornis auricularis* & miudinho & 8 & SUB & INS & $\mathrm{B}$ \\
\hline Euscarthmus meloryphus & barulhento & 1 & $\mathrm{COP}$ & INS & B \\
\hline Pitangus sulphuratus & bem-te-vi & 8 & $\mathrm{COP}$ & FRU/INS & $\mathrm{B}$ \\
\hline Myiodynastes maculatus & bem-te-vi-rajado & 5 & $\mathrm{COP}$ & FRU/INS & B \\
\hline Myiozetetes similis & bentevizinho-de-penacho-vermelho & 2 & $\mathrm{COP}$ & FRU/INS & $\mathrm{B}$ \\
\hline Empidonomus varius & peitica & 5 & $\mathrm{COP}$ & FRU/INS & B \\
\hline Lathrotriccus euleri & enferrujado & 12 & SUB & FRU/INS & M \\
\hline Vireo chivi & juruviara & 13 & COP & INS & $\mathrm{B}$ \\
\hline Turdus leucomelas & sabiá-branco & 12 & $\mathrm{SUB} / \mathrm{COP}$ & FRU/INS & $\mathrm{B}$ \\
\hline Turdus rufiventris & sabiá-laranjeira & 14 & $\mathrm{SUB} / \mathrm{COP}$ & FRU/INS & $\mathrm{B}$ \\
\hline Turdus amaurochalinus & sabiá-poca & 4 & SUB/COP & FRU/INS & $\mathrm{B}$ \\
\hline Basileuterus culicivorus & pula-pula & 18 & SUB & INS & M \\
\hline Tangara cyanoventris* & saíra-douradinha & 4 & $\mathrm{COP}$ & FRU & M \\
\hline Tangara sayaca & sanhaçu-cinzento & 15 & $\mathrm{COP}$ & FRU & B \\
\hline Conirostrum speciosum & figurinha-de-rabo-castanho & 2 & $\mathrm{COP}$ & FRU/INS & B \\
\hline Hemithraupis ruficapilla* & saíra-ferrugem & 1 & $\mathrm{COP}$ & FRU/INS & B \\
\hline Tersina viridis & saí-andorinha & 9 & $\mathrm{COP}$ & FRU & $\mathrm{B}$ \\
\hline Habia rubica & tiê-do-mato-grosso & 3 & SUB & INS & A \\
\hline
\end{tabular}

Neste contexto, a presença desse grupo pode ser considerada como indicadora de boa qualidade do habitat estudado, indicando que a floresta restaurada encontra-se em estádio avançado de sucessão, corroborando os estudos fitossociológicos realizados por Miranda Neto et al. (2012a; 2012b). 
TABELA 2: Número de espécies (\%) e número de contatos (\%) das espécies de aves em cada categoria avaliado em trecho de floresta restaurado em Minas Gerais.

TABLE 2: $\quad$ Bird species (\%) and number of contacts (\%) recorded in a 40-year old restored forest in Minas Gerais state, considering the feeding behavior, the feeding habits and the sensitivity to disturbance.

\begin{tabular}{lcc}
\hline Categorias & Espécies (\%) & Contatos (\%) \\
\hline Hábitos alimentares: & & \\
Insetívoros & $14(45,2 \%)$ & $90(44,1 \%)$ \\
Frugívoros-Insetívoros & $12(38,7 \%)$ & $79(38,7 \%)$ \\
Frugívoros & $5(16,1 \%)$ & $35(17,2 \%)$ \\
Estrato de forrageio e nidificação: & & \\
Copa & $18(58,1 \%)$ & $97(47,5 \%)$ \\
Sub-bosque & $13(41,9 \%)$ & $107(52,5 \%)$ \\
Sensibilidade: & & \\
Alta & $1(3,2 \%)$ & $3(1,5 \%)$ \\
Média & $10(32,3 \%)$ & $83(40,7 \%)$ \\
Baixa & $20(64,5 \%)$ & $118(57,8 \%)$ \\
\hline
\end{tabular}

Entre as aves de copa, as frugívoras/insetívoras e as frugívoras, foram as mais representativas em número de espécies, com $39 \%$ e $17 \%$, respectivamente do total de espécies deste grupo, com destaque para a abundância de importantes dispersores de sementes, como Tangara sayaca e Turdus rufiventris (PIZO, 2004) (Tabelas 1 e 2). Em florestas em restauração, a presença de fauna consumidora de frutos e sementes é apontada como positiva para o processo de sucessão florestal, por sua capacidade de dispersão de sementes e, portanto, por contribuírem com os processos de regeneração natural e enriquecimento (PARROTA; TURNBULL; JONES, 1997; VOLPATO et al., 2012), podendo ser assim utilizados como bons indicadores do sucesso de projetos de restauração. Miranda Neto et al. (2012b) observaram que a composição florística dessa floresta restaurada foi formando principalmente por espécies com dispersão zoocóricas, que ultrapassou $40 \%$ em relação às outras síndromes de dispersão. Assim, a alta proporção de espécies de aves consumidoras de frutos era esperada e reflete as características florísticas da área. Contudo, deve-se ressaltar que, entre as espécies arbóreas com maior densidade registrada por Miranda Neto et al. (2012b) neste trecho de floresta restaurada está a palmeira-australiana Archontophoenix cunninghamiana H. Wendl. e Drude com apenas cinco indivíduos plantados durante o processo de reflorestamento da área. No presente estudo foram observadas três espécies de aves se alimentando dos frutos maduros de Archontophoenix cunninghamiana: Penelope obscura, Tersina viridis e Tangara sayaca. Christianini (2006) observou seis espécies de aves consumindo os frutos maduros de Archontophoenix cunninghamiana, sendo que as espécies que mais consumiram os frutos foram Pitangus sulphuratus e Turdus rufiventris, ambas registradas no presente estudo. Esta palmeira por ser considerada uma espécie invasora e bastante agressiva (DISLICH; KISSER; PIVELLO, 2002; CHRISTIANINI, 2006), a sua alta ocorrência e o seu consumo e dispersão de propágulos por aves evidencia sua ameaça às áreas de florestas mais conservadas (MIRANDA NETO et al. 2012b). Neste contexto, a remoção dos indivíduos adultos pode ser uma alternativa para reduzir a invasão desta espécie exótica na área.

A busca por alternativas viáveis para a avaliação da restauração florestal é constante. Em projetos de restauração no domínio da Floresta Atlântica, o monitoramento e a avaliação do sucesso da restauração são feitos considerando-se a estrutura da vegetação e na diversidade de espécies de plantas, sendo ainda escasso o uso de espécies da fauna (RODRIGUES et al., 2009). Volpato e Martins (2013) estudando uma área em restauração com 10 anos de implantação, a partir de plantio de Acacia sp., registraram um total de 33 espécies de aves ao longo de dois anos de levantamento. Apesar do número de espécies registrado por Volpato e Martins (2013) ser muito semelhante ao apresentado no presente estudo, uma comparação da composição das espécies, revela que as comunidades de aves foram distintas: no plantio de exótico houve o predomínio de espécies de aves tipicamente de áreas abertas e granívoras, como consequência da ausência da regeneração do sub-bosque e domínio de gramíneas (Setaria vulpiseta (Lam.) Roem. \& Schult). 
Outro detalhe importante destacado no presente estudo, e que fica evidente pela comparação com o estudo de Volpato e Martins (2013) e que nem sempre a riqueza de espécies (i.e. o número de espécies) pode ser um bom parâmetro para avaliar o sucesso de restauração de uma área. Dessa maneira, o conhecimento das características ecológicas das espécies de aves torna-se fundamental para se utilizar as aves com indicadores de avaliação na restauração ecológica de áreas degradadas (KRITZINGER; VAN AARDE, 1998; PADOVEZI; RODRIGUES; HORBACH, 2014).

Um dos grandes desafios da restauração a partir do reflorestamento tradicional é evitar a formação de "florestas vazias", caracterizadas por uma baixa ou mesmo ausência de fauna em consequência da falta de estratificação vertical e diversidade de habitat (MARTINS; MIRANDA NETO; RIBEIRO, 2012). Contudo, Miranda Neto et al. (2012a) descreve esse trecho de floresta restaurado como uma área com alta diversidade de estratos e grande número de árvores de grande porte (atingido 27,2 $\mathrm{m}$ de altura). Miranda Neto et al. (2012b) também observaram que $49 \%$ das espécies registradas do estrato arbustivo/arbóreo não foram oriundas do plantio realizado há 40 anos, mas providentes de remanescentes florestais presentes no entorno. Esses autores argumentam que a localização desse trecho em uma matriz florestal foi fundamental para esta floresta atingir um estádio avançado de sucessão, contribuindo para a diversidade florística e atração da fauna, incluindo as dispersoras de sementes. Além disso, a presença de uma matriz florestal contribuiu especialmente para a presença das aves de sub-bosque, que são intolerantes a ambientes abertos e com baixa capacidade de dispersão entre fragmentos florestais isolados (CANADAY, 1997).

\section{CONCLUSÃO}

Apesar da baixa riqueza de espécies amostradas, o trecho de floresta restaurada com 40 anos apresenta uma comunidade de aves tipicamente da Floresta Estacional Semidecidual do domínio da Mata Atlântica. A presença de aves consumidoras e dispersoras de sementes e de espécies de sub-bosque nesta floresta restaurada pode ser um indicativo de que a área se encontra em um estágio de sucessão autossustentável, necessitando intervenção de manejo florestal apenas para a erradicação da palmeira invasora Archontophoenix cunninghamiana.

Os resultados do presente estudo também demonstram que mesmo em uma área pequena e com poucos dias amostragens é possível obter uma caracterização da comunidade de aves e, a partir dessa composição, relacioná-la com as condições do habitat, permitindo uma avaliação rápida e segura do sucesso de um projeto de restauração florestal, e, portanto, mostra que a avifauna pode ser um importante indicador para determinar o estado atual de um projeto de restauração florestal.

\section{AGRADECIMENTO}

O primeiro autor agradece a FAPEMIG pela bolsa de pós-doc júnior concedida durante o trabalho e o segundo autor agradece ao CNPq pela bolsa de produtividade.

\section{REFERÊNCIAS}

ALEIXO, A. Conservação da avifauna da Floresta Atlântica: efeitos da fragmentação e a importância de florestas secundárias. In: ALBUQUERQUE, J. L. B. et al. (Ed.). Ornitologia e conservação: da ciência às estratégias. Tubarão: Unisul, 2001. p. 199-206.

ALEIXO, A.; VIELLIARD, J. M. E. Composição e dinâmica da comunidade de aves da Mata de Santa Genebra, Campinas, SP. Revista Brasileira de Zoologia, Curitiba, v. 12, n. 3, p. 493-511, 1995.

ANJOS, L. Bird communities in five Atlantic forest fragments in southern Brazil. Ornitologia Neotropical, St. Louis, v. 12, n. 1, p. 11-27, 2001.

ANJOS, L. A eficiência do método de amostragem por pontos de escuta na avaliação da riqueza de aves. Revista Brasileira de Ornitologia, São Paulo, n. 15, v. 2, p. 239-243, 2007.

ANTUNES, A. Z. Riqueza e dinâmica de aves endêmicas da Mata Atlântica em um fragmento de floresta estacional semidecidual no sudeste do Brasil. Revista Brasileira de Ornitologia, São Paulo, v. 15, n. 1, p. 61-68, 2007. 
ARATO, H. D.; MARTINS, S. V.; FERRARI, S. H. S. Produção e decomposição de serapilheira em um sistema agroflorestal implantado para recuperação de área degradada em Viçosa- MG. Revista Árvore, Viçosa, MG, v. 27, n. 5, p. 715-721, 2003.

ARAÚJO, F. S. et al. Estrutura da vegetação arbustivo-arbórea colonizadora de uma área degradada por mineração de caulim, Brás Pires, MG. Revista Árvore, Viçosa, MG, v. 30, n. 1, p. 107-116, 2006.

ARAÚJO, F. S. et al. Florística da vegetação arbustivo - arbórea colonizadora de uma área degradada por mineração de caulim, em Brás Pires, MG. Revista Árvore, Viçosa, MG, v. 29, n. 6, p. 983-992, 2005.

BENCKE G. A. et al. Áreas importantes para a conservação das aves no Brasil. Parte I - Estados do domínio da Mata Atlântica. São Paulo: SAVE Brasil, 2006.

BIBBY, C. J.; BURGESS, N. D.; HILL, D. A. Bird census techniques. London: Academic Press, 1992.

BROOKS, T. M.; BALMFORD, A. Atlantic Forest extinctions. Nature, London, n. 380, v. 6570, p. 115115, 1996.

CANADAY, C. Loss of insectivorous birds along gradient of human impact in Amazonia. Biological Conservation, Cambridge, v. 77, n. 1, 63-77, 1997.

CHRISTIANINI, A. V. Fecundidade, dispersão e predação de sementes de Archontophoenix cunninghamiana H. Wendl. \& Drude, uma palmeira invasora da Mata Atlântica. Revista Brasileira de Botânica, São Paulo, v. 29 , n. 4 , p. 587-594, 2006.

COLWELL, R. K. EstimateS: statistical estimation of species richness and shared species from samples. Version 8.0.0. User's Guide and application published. [s. 1.: s. n.], 2006. Disponível em: $<$ http://purl.oclc.org/estimates $>$.

DISLICH, R.; KISSER, N.; PIVELLO, V. R. A invasão de um fragmento florestal em São Paulo (SP) pela palmeira australiana Archontophoenix cunninghamiana H. Wendl. e Drude. Revista Brasileira de Botânica, São Paulo, v. 25, n. 1, p. 55-64, 2002.

DURIGAN, G.; ENGEL, V. L. Restauração de ecossistemas no Brasil: onde estamos e para onde podemos ir? In: MARTINS, S. V. (Ed.). Restauração ecológica de ecossistemas degradados. Viçosa, MG: Editora UFV, 2012. p. 41-68.

KRITZINGER. J. J.; VAN AARDE, R. J. The bird communities of rehabilitating coastal dunes at Richards Bay, KwaZulu-Natal. South African Journal of Science, Johannesburg, v. 94, n. 71-78, 1998.

LAWTON, J. H. Population abundance, geographic range and conservation: 1994 Witherby Lecture. Bird Study, Londres, v. 43, n. 1, p. 3-19, 1996.

MARISCAL-FLORES, E. J. Potencial produtivo e alternativas de manejo sustentável de um fragmento de Mata Atlântica secundária, Município de Viçosa, Minas Gerais. 1993. 165 f. Dissertação (Mestrado em Ciência Florestal) - Universidade Federal de Viçosa, Viçosa, MG, 1993.

MARTINS, S. V.; MIRANDA NETO, A.; RIBEIRO, T. M. Uma abordagem sobre a diversidade e técnicas de restauração ecológica. In: MARTINS, S. V. Restauração Ecológica de Ecossistemas Degradados. Viçosa, MG: Editora UFV, 2012. p. 17-40.

MARTINS, S. V. et al. Banco de sementes como indicador de restauração de uma área degradada por mineração de caulim em Brás Pires, MG. Revista Árvore, Viçosa, MG, v. 32, n. 6, p. 1081-1088, 2008.

MARTINS, S. V. Recuperação de áreas degradadas: ações em áreas de preservação permanentes, voçorocas, taludes rodoviários e de mineração. Viçosa, MG: Aprenda Fácil, 2009a.

MARTINS, S. V. Soil seed bank as indicator of forest regeneration potential in canopy gaps of a semideciduous forest in Southeastern Brazil. In: FOURNIER, M. V. Forest regeneration: ecology, management and economics. New York: Nova Science Publishers, 2009b. p. 113-128.

MEIRA-NETO, J. A. A.; MARTINS, F. R. Composição florística de uma Floresta Estacional Semidecidual Montana no município de Viçosa-MG. Revista Árvore, Viçosa, MG, v. 26, n. 4, p. 437-446, 2002.

MELO, A. C. G.; MIRANDA, D. L. C.; DURIGAN, G. Cobertura de copas como indicador de desenvolvimento estrutural de reflorestamentos de restauração de matas ciliares no Médio Vale do Paranapanema, SP, Brasil. Revista Árvore, Viçosa, MG, v. 31, n. 2, p. 312-328, 2007.

MIRANDA NETO, A. et al. Estrato de regeneração natural de uma floresta restaurada com 40 anos. Pesquisa Florestal Brasileira, Colombo, v. 32, n. 72, p. 409-420, 2012a.

MIRANDA NETO, A. et al. Florística e estrutura do estrato arbustivo-arbóreo de uma floresta restaurada com 40 anos, Viçosa, MG. Revista Árvore, Viçosa, MG, v. 36, n. 5, p. 869-878, 2012 b. 
MUNRO, N. T. et al. Revegetation in agricultural areas: the development of structural complexity and floristic diversity. Ecological Applications, Washington, v. 19, n. 5, p. 1197-1210, 2009.

MUNRO, N. T. et al. Bird's response to revegetation of different structure and floristics. Are restoration plantings restoring bird communities? Restoration Ecology, Washington, v. 19, n. 201, p. 223-235, 2010. NICHOLS, O. G.; GRANT, C. D. Vertebrate fauna recolonization of restored bauxite mines-key findings from almost 30 years of monitoring and research. Restoration Ecology, Washington, v. 15, n. 4 supl, p. 116-126, 2007.

PADOVEZI, A.; RODRIGUES, R. R.; HORBACH, M. A. Avifauna como possível indicador da resiliência de áreas degradadas. Advances in Forestry Science, Cuiabá, v. 1, n. 1, p. 11-17, 2014.

PARKER, T. A.; STOTZ, D. E.; FITZPATRICK, J. W. Ecological and distribution databases. In: STOTZ, D. E. et al. (Eds.). Neotropical birds: ecology and conservation. Illinois: University of Chicago Press, 1996. p. 131-436.

PARROTTA, J. A.; TURNBULL, J. W.; JONES, N. Catalyzing native forest regeneration on degraded tropical lands. Forest Ecology and Management, Amsterdam, v. 99, n. 1, p. 1-7, 1997.

PIACENTINI, V. Q. et al. Annotated checklist of the birds of Brazil by the Brazilian Ornithological Records Committee. Revista Brasileira de Ornitologia, Belém, v. 23, n. 2, p. 91-298, 2015.

PIMM, S. L. et al. The future of biodiversity. Science, Washington, v. 269, n. 5222, p. 347-350, 1995.

PIZO, M. A. Frugivory and habitat use by fruit-eating birds in a fragmented landscape in southeast Brazil.

Ornitologia Neotropical, Montreal, v. 15, n. 1 supl, p. 117-126, 2004.

RIBON, R.; SIMON, J. E.; DE MATTOS, G. T. Bird extinctions in Atlantic Forest Fragments of Viçosa Region, Southeastern Brazil. Conservation Biology, Malden, v. 17, n. 6, p. 1827-1839, 2003.

RODRIGUES, R. R. et al. On the restoration of high diversity forests: 30 years of experience in Brazilian Atlantic Forest. Biological Conservation, Essex, v. 142, n. 6, p. 1242-1251, 2009.

VOLPATO, G. H. et al. O Papel ecológico das aves dispersoras de sementes na restauração ecológica. In: MARTINS, S. V. Restauração ecológica de ecossistemas degradados. Viçosa, MG: Editora UFV, 2012. p. 191-211.

VOLPATO, G. H. et al. Terrestrial passerines in an Atlantic Forest remnant of southern Brazil. Brazilian Journal of Biology, Rio de Janeiro, v. 66, n. 2A, p. 473-478, 2006.

VOLPATO, G. H. et al. The use of the point count method for bird survey in the Atlantic forest. Zoologia, Curitiba, n. 26, v. 1, p. 74-78, 2009.

VOLPATO, G. H.; MARTINS, S. V. The bird community in naturally regenerating Myracrodruon urundeuva (Anacardiaceae) forest in southeastern Brazil. Revista de Biologia Tropical, Costa Rica, n. 61, v. 4, p. 1585-1595, 2013.

VOLPATO, G. H.; PRADO, V. M.; ANJOS, L. What can tree plantations do for forest birds in fragmented forest landscapes? A case study in southern Brazil. Forest Ecology and Management, Amsterdam, v. 260, n. 7 , p. 1156-1163, 2010. 